$17^{\text {th }}$ International Congress of Metrology, 14001 (2015)

DOI: $10.1051 /$ metrology / 201514001

(C) Owned by the authors, published by EDP Sciences, 2015

\title{
Metrology for Graphene and 2-D Materials
}

\author{
Andrew J. Pollard ${ }^{1}$ \\ ${ }^{1}$ National Physical Laboratory, Hampton Road, Teddington, TW11 OLW, United Kingdom
}

\begin{abstract}
The application of graphene, a one atom-thick honeycomb lattice of carbon atoms with superlative properties, such as electrical conductivity, thermal conductivity and strength, has already shown that it can be used to benefit metrology itself as a new quantum standard for resistance. However, there are many application areas where graphene and other 2-D materials, such as molybdenum disulphide $\left(\mathrm{MoS}_{2}\right)$ and hexagonal boron nitride (h-BN), may be disruptive, areas such as flexible electronics, nanocomposites, sensing and energy storage. Applying metrology to the area of graphene is now paramount to enable the emerging global graphene industry and bridge the gap between academia and industry. Measurement capabilities and expertise in a wide range of scientific areas are required to address this challenge. The combined and complementary approach of varied characterisation methods for structural, chemical, electrical and other properties, will allow the real-world issues of commercialising graphene and other 2-D materials to be addressed. Here, examples of metrology challenges that have been overcome through a multi-technique approach are discussed. In addition, the role of international standardisation in this area is described, outlining the current work ongoing in both the International Organization of Standardization (ISO) and the International Electrotechnical Commission (IEC).
\end{abstract}

\section{Introduction}

Since its first isolation in 2004 [1], the level of scientific interest in graphene has increased exponentially, an increase that shows no sign of slowing since the award of the 2010 Nobel Prize in Physics to Geim and Novoselov. The reason for this level of interest is the wide range of extraordinary properties of this first 2-D material, such as its electrical conductivity, mechanical strength, optical transparency and thermal properties. Thus, this one-atom thick honeycomb lattice of carbon atoms promises to have an impact on many different industry application areas, ranging from composite materials to biological sensors [2].

One of graphene's greatest accomplishments may actually be initiating the new field of 2-D materials, including layered materials such as molybdenum disulphide and hexagonal boron nitride, which offer equally interesting but different properties, such as semiconducting, superconducting or insulating materials with a nanoscale thickness, that can be selected as part of a '2-D material toolbox'. This potential has not gone unnoticed by industry and governments alike, with the European Commission announcing the EU Graphene Flagship project in 2012, boasting one billion Euros investment over a 10 year period.

In the field of metrology, graphene has already proven itself particularly impressive as a quantum resistance standard [3] demonstrating a quantum Hall resistance quantization accuracy of three parts per billion. However, the field of graphene is now ready for metrology to repay the favour, as an enabler of the commercialisation of these 2-D materials as they begin their uptake into industry; there are now reported to be several hundred companies worldwide producing or using graphene. Despite this level of industry involvement, there are many measurement issues that need to be addressed for this nanoscale material, which brings with it many challenges. A vast array of metrology research into structural, chemical, electrical, thermal and mechanical properties will be required to bridge the 'valley of death' in the commercialisation cycle that graphene now faces, as every new technology does.

As the UK's National Measurement Institute, the National Physical Laboratory (NPL) aims to continue to advance the metrology science for graphene and 2-D materials, addressing the gaps in characterisation understanding and enabling the international standardisation of this area. In particular, the reproducible and accurate measurement of the different properties of these 2-D materials themselves, but also the measurement of these materials when used in the more near-term industry areas, such as nanocomposites, printed and flexible electronics and sensing applications. To this end, the determination of the structural and chemical properties of the materials themselves and thus the understanding of the nanoscale ingredients affecting the properties of the macroscale products is the first step in any application development process.

\section{Characterisation of Graphene Defects}

The level of disorder in graphene, that is, how defective graphene is, adversely affects its properties. It is important to note that the terminology of graphene defects is specific and is not the same as a macroscale defect that may be described for consumer products. Graphene defects are specific to graphene and on the atomic-scale, typically being a vacancy defect (missing

Email corresponding author: andrew.pollard $@$ npl.co.uk 
atom or atoms) or a dislocation defect (atoms that are not positioned in a continuous honeycomb lattice). The latter may be in the form of a point defect or a line defect, the latter being the case for a grain boundary commonly found in chemical vapour deposition (CVD) grown graphene.

Commonly, the presence of defects has a negative perception, as an increased defect density is known to deteriorate the electronic properties of graphene [4]. However, defects may also enhance the properties of graphene in other ways, an example being the application area of membranes for filtering of ionic species [5]. For this application area, vacancy defects are required for the material to be viable. This shows that for any application area, although defects may or may not be desired, the measurement of the level of disorder is a definite requirement.

Although techniques such as scanning tunnelling microscopy (STM) and transmission electron microscopy (TEM) can be used to directly visualise the defects within a graphene layer, these techniques are not high-throughput methods for determining the level of disorder. However, Raman spectroscopy can be performed on graphene layers quickly and nondestructively whilst providing information on the density of defects. This has been investigated previously [6,7] and a relationship determined between the ratio of the Raman D- and G-peak and the inter-defect distance, $L_{\mathrm{D}}$, as shown in Equation 1:

$$
\frac{I_{\mathrm{D}}}{I_{\mathrm{G}}}=C_{\mathrm{A}} \frac{\left(r_{\mathrm{A}}^{2}-r_{\mathrm{S}}^{2}\right)}{\left(r_{\mathrm{A}}^{2}-2 r_{\mathrm{S}}^{2}\right)}\left[e^{-\pi r_{\mathrm{S}}^{2} / L_{\mathrm{D}}^{2}}-e^{-\pi\left(r_{\mathrm{A}}^{2}-r_{\mathrm{S}}^{2}\right) / L_{\mathrm{D}}^{2}}\right]
$$

$C_{\mathrm{A}}$ is a constant related to the wavelength of the Raman excitation laser, $r_{\mathrm{A}}$ is a variable related by $L_{\sigma}=r_{\mathrm{S}}+r_{\mathrm{A}}$, where $L_{\sigma}$ is the distance travelled by the photoexcited electron-hole pair. $r_{\mathrm{S}}$ is the average radius of the defects contained within the area probed by the Raman spectroscopy measurement (typically hundreds of nanometres in diameter). However, measurements to identify the relationship between the Raman peak intensity ratio or defect density with the defect size were required.

Therefore, we introduced vacancy defects of different sizes into single-layer graphene flakes, produced through mechanical exfoliation, and studied changes in the Raman D- and G-peaks with varying defect densities [8]. To produce well-controlled defect densities and sizes three different types of ion guns of a secondary ion mass spectrometry (SIMS) instrument were used; $\mathrm{Mn}^{+}, \mathrm{Bi}^{+}$ and $\mathrm{Bi}_{3}{ }^{+}$at $25 \mathrm{keV}$. To determine the values of $r_{\mathrm{S}}$ for each type of ion a highly orientated pyrolytic graphite (HOPG) sample was bombarded and STM measurements were performed on 20 different defects created by each type of ion, as shown in Figure 1. Values of $r_{\mathrm{S}}$ of $0.6 \pm 0.1 \mathrm{~nm}$, $1.3 \pm 0.1 \mathrm{~nm}$ and $1.9 \pm 0.1 \mathrm{~nm}$ were found for $\mathrm{Mn}^{+}, \mathrm{Bi}^{+}$ and $\mathrm{Bi}_{3}{ }^{+}$respectively. (a)

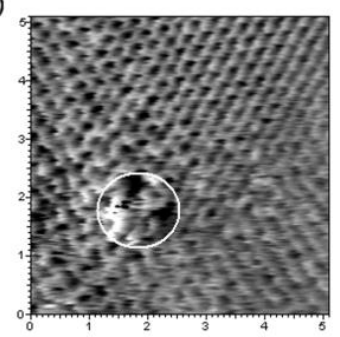

(c)
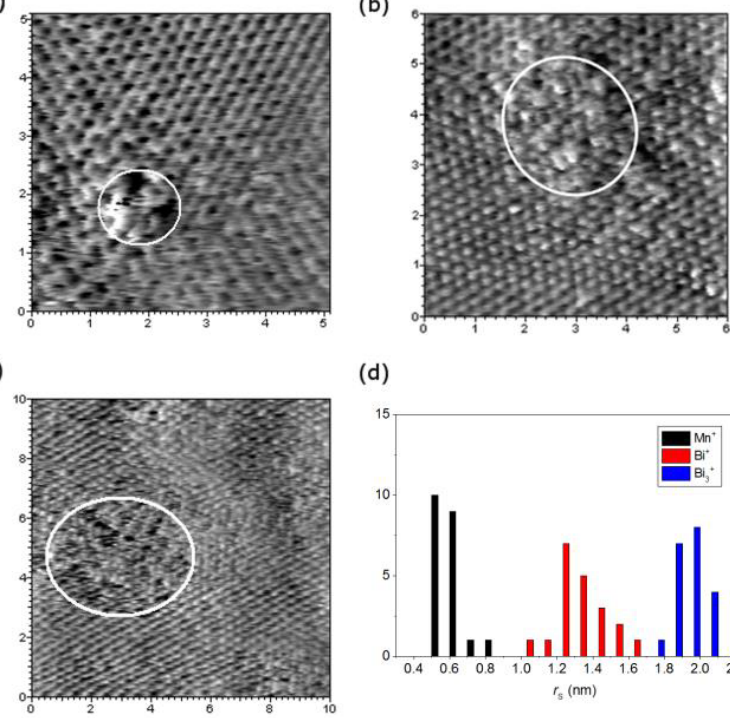

(d)

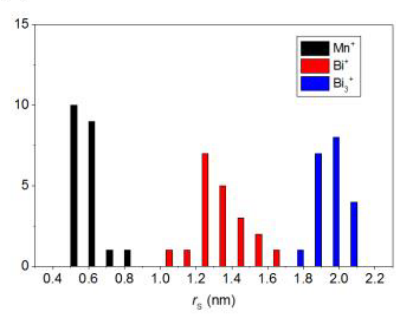

Figure 1. Example scanning tunneling microscopy (STM) images of disordered areas of HOPG (graphite) after ion bombardment by (a) $\mathrm{Mn}^{+}$, (b) $\mathrm{Bi}^{+}$and (c) $\mathrm{Bi}_{3}{ }^{+}$ions at $25 \mathrm{keV}$, (sample voltage $-50 \mathrm{mV}$, tunnel current $1.0 \mathrm{nA}$, scale in $\mathrm{nm}$ ), a white ellipse for each STM image illustrates the determined disordered area of the HOPG lattice. (d) Histogram of values of $r_{\mathrm{s}}$ for the three different ion types, from [8].

The evolution of Raman spectra obtained with a $532 \mathrm{~nm}$ excitation wavelength of single-layer graphene (1LG) in Figure $2 \mathrm{a}$ shows an increase in the $I_{\mathrm{D}} / I_{\mathrm{G}}$ ratio with increasing defect density (decreasing $L_{\mathrm{D}}$ ) until Stage 2 disorder occurs, as observed for $L_{\mathrm{D}}=0.9$. Then the Raman peaks start to broaden and the $I_{\mathrm{D}} / I_{\mathrm{G}}$ ratio decreases, an evolution observed for all three types of defects. No Dpeak is observed for pristine graphene as this Raman peak requires a defect for its activation. The increase in the D-peak width observed in Figure $2 \mathrm{~b}$ is staggered with defect size, that is, a larger value of $r_{\mathrm{S}}$ leads to Stage 2 disorder at a larger value of $L_{\mathrm{D}}$.

Figure 2c shows a pronounced difference in the behaviour of the Raman $I_{\mathrm{D}} / I_{\mathrm{G}}$ ratio for different size defects caused by different types of ion bombardment. An increase in the $I_{\mathrm{D}} / I_{\mathrm{G}}$ ratio is observed as the defect density increases ( $L_{\mathrm{D}}$ decreases) until a maximum is reached at the interface between Stage 1 and Stage 2 disorder. The $I_{\mathrm{D}} / I_{\mathrm{G}}$ ratio then decreases again with increasing defect density, as expected from the Raman spectra in Figure 2a, for all three types of vacancy defect. However, the quantitative variation in $I_{\mathrm{D}} / I_{\mathrm{G}}$ is different for each type of defect. As observed in Figure 2b, the change from Stage 1 to Stage 2 disorder occurs at different values of $L_{\mathrm{D}}$ depending on the type of ion bombardment and thus the size of the defect. The maximum value of $I_{\mathrm{D}} / I_{\mathrm{G}}$ is also different, with larger values for smaller values of $r_{\mathrm{s}}$. However, using the values of $r_{\mathrm{S}}$ determined through STM measurements, Equation 1 can be calculated for each set of data, revealing a good fit of the experimental data for values of $C_{\mathrm{A}}=5.0 \pm 0.9$ and $L_{\sigma}=2.4 \pm 0.6 \mathrm{~nm}$, shown in Figure $2 \mathrm{c}$. 
(a)

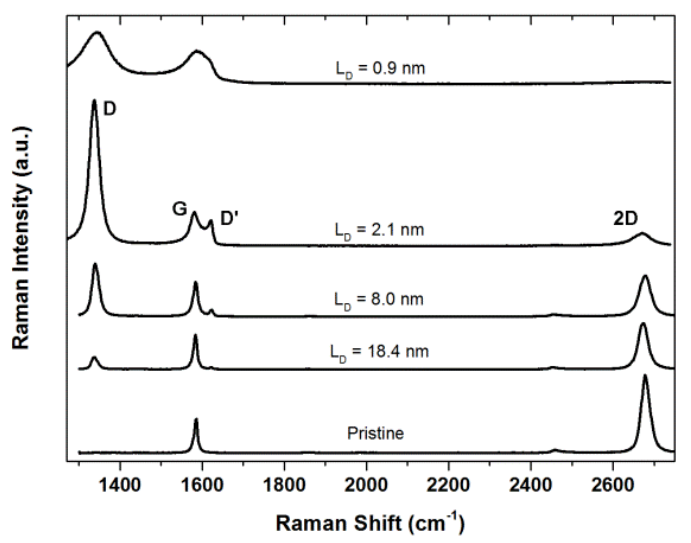

(b)

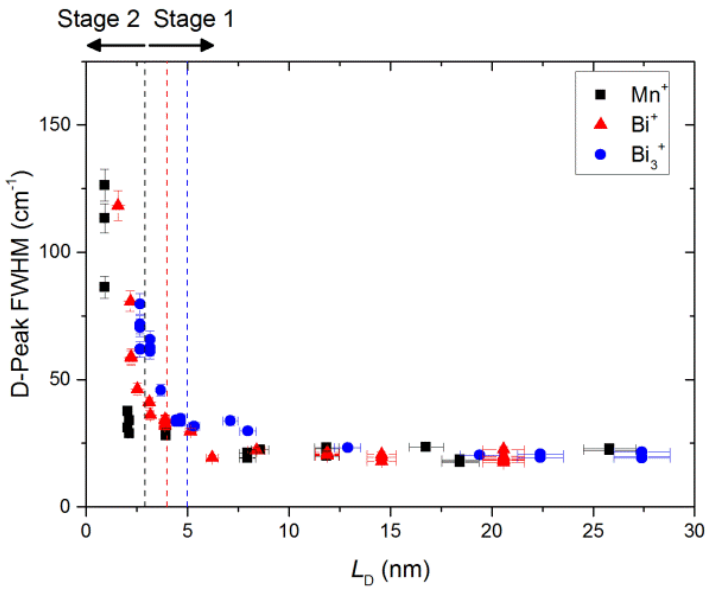

(c)

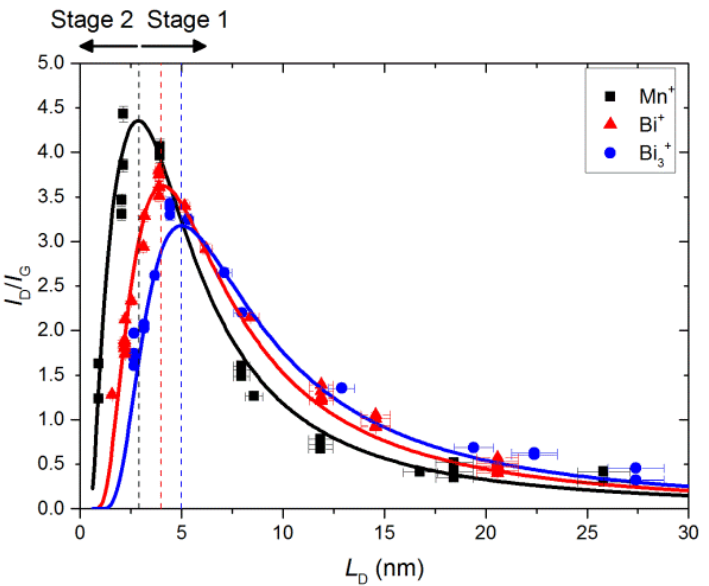

Figure 2. (a) Raman spectra obtained for $1 \mathrm{LG}$ bombarded with $\mathrm{Mn}^{+}$. The evolution of the D-peak is clearly shown for decreasing $L_{\mathrm{D}}$. Spectra are shown offset vertically for illustrative purposes. (b) D-peak full width at half-maximum (FWHM) versus the average inter-defect distance, $L_{\mathrm{D}}$ and (c) D-peak to G-peak ratio $\left(I_{\mathrm{D}} / I_{\mathrm{G}}\right)$ versus $L \mathrm{D}$, for $\mathrm{Mn}^{+}$(black squares), $\mathrm{Bi}^{+}$(red triangles) and $\mathrm{Bi}^{+}$(blue circles) ion bombardment of $1 \mathrm{LG}$. The approximate transition point from Stage 1 to Stage 2 is denoted in (b) and (c) by a dashed vertical line and colored according to the ion bombardment, for the specific case of the dashed black line for $\mathrm{Mn}^{+}$ions, Stage 1 and Stage 2 are annotated. Eqn. 1 is fitted to the data in (c) for different values of $r$ s for different ions as determined with STM, from [8].

This work from reference [8] shows experimentally that the $I_{\mathrm{D}} / I_{\mathrm{G}}$ ratio is affected by the size of point defects. This means that Raman spectroscopy is not only a powerful technique for determining the defect density in graphene, but also for determining the defect size. However, to quantitatively determine either the defect density or the defect size with solely Raman spectroscopy, the other variable must first be determined using a complementary method. This work has also enabled a decrease in the uncertainty in $C_{\mathrm{A}}$ and $L_{\sigma}$ for graphene, which will allow improved measurements for future Raman spectroscopy characterisation of defects within graphene.

\section{Characterisation of $\mathrm{MoS}_{2}$ Defects}

Graphene has now had over a decade of research from which a vast scientific knowledge base has been established. However, the many other 2-D materials that are now being explored [9] have had far fewer investigations into the characterisation of their properties. $\mathrm{MoS}_{2}$ is one of these less explored 2-D materials but has been gathering a significant interest due to its semiconducting properties.
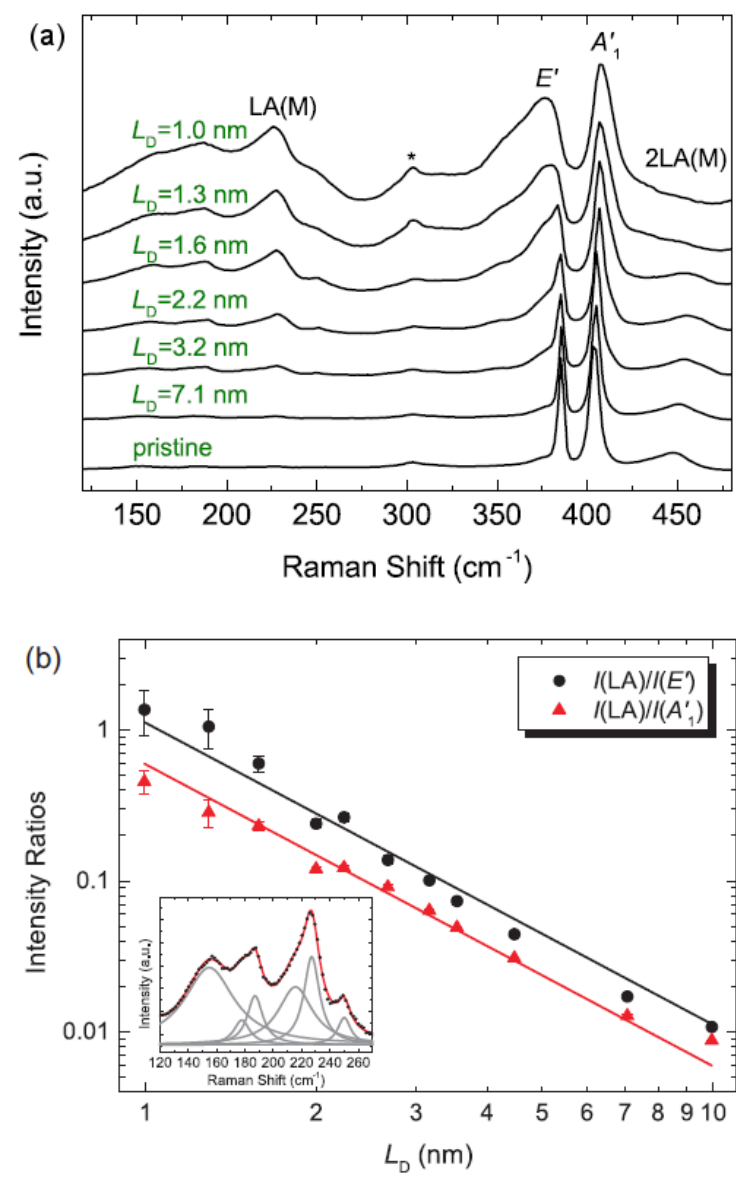

Figure 3. (a) Raman spectra of $1 \mathrm{~L}-\mathrm{MoS}_{2}$ flakes with varying inter-defect distances $L_{\mathrm{D}}$. The asterisk refers to the $2 \mathrm{TA}(X)$ Raman peak of the Si substrate. The spectra have been normalised to the intensity of the $A$ ' 1 peak, as observed from the relative increase of $2 \mathrm{TA}(X)$ with decreasing $L_{\mathrm{D}}$. (b) Experimental intensity ratios $I(\mathrm{LA}) / I\left(A^{\prime}{ }_{l}\right)$ and $I(\mathrm{LA}) / I\left(E^{\prime}\right)$ are reported in symbols. The error bars represent the standard error from the fitting process. The solid lines are linear fits with slope equal to -2 . The low frequency bands, where the LA peak is located at $\sim 227 \mathrm{~cm}^{-1}$, are shown in the inset, along with the corresponding Lorentzian fits, from [10]. Copyright (2015) by the American Physical Society. 
Although $\mathrm{MoS}_{2}$ has been used in many application areas for several decades, it has only been exfoliated down to single-layer $\mathrm{MoS}_{2}\left(1 \mathrm{~L}-\mathrm{MoS}_{2}\right)$ more recently [11]. $\mathrm{MoS}_{2}$ does not have the same honeycomb structure as graphene or h-BN layers, with each Mo atom bonded to six sulphur atoms in a trigonal prismatic geometry, forming a 2-D material that can be thought of as a layer of Mo atoms sandwiched between two layers of sulphur atoms. When $\mathrm{MoS}_{2}$ is exfoliated down to a single-layer it becomes a direct band-gap semiconducting material rather than the indirect semiconducting material of its bulk form.

Raman spectroscopy measurements of $\mathrm{MoS}_{2}$ understandably produce very different spectra to comparable measurements of graphene, and the systematic investigation of Raman spectra of $1 \mathrm{~L}-\mathrm{MoS}_{2}$ with varying levels of defects introduced has not been undertaken until recently [10]. Mechanically exfoliated $1 \mathrm{~L}-\mathrm{MoS}_{2}$ was bombarded with $\mathrm{Mn}^{+}$as previously described for graphene layers, producing $1 \mathrm{~L}-\mathrm{MoS}_{2}$ flakes with known defect densities. Spectra from subsequent Raman spectroscopy measurements are shown in Figure $3 \mathrm{a}$ and show the evolution of the spectra with defect density. Although there are changes in many different peaks for this material, of particular note is a previously uninvestigated Raman peak for $1 \mathrm{~L}-\mathrm{MoS}_{2}$, at $\sim 227 \mathrm{~cm}^{-1}$, which is attributed to the longitudinal acoustic (LA) branch at the edge of the Brillouin zone for phonons with momentum $\mathbf{q} \neq 0$ at the $\mathbf{M}$-point. Calculating the ratio of this LA(M) peak with the first-order $E^{\prime}$ and $A_{1}^{\prime}$ Raman peaks at $\sim 382 \mathrm{~cm}^{-1}$ and $\sim 407 \mathrm{~cm}^{-1}$ respectively, a trend is observed for changes in $L_{\mathrm{D}}$, as shown in Figure $3 \mathrm{~b}$. The LA(M) Raman peak at $\sim 227 \mathrm{~cm}^{-1}$ for $1 \mathrm{~L}-\mathrm{MoS}_{2}$ is therefore analogous to the D-peak for graphene, and can be used to quantitatively determine the defect density in $\mathrm{MoS}_{2}$ by normalising the intensity to either the $E^{\prime}$ and $A^{\prime}{ }_{1}$ Raman peaks [10].

New peaks are not the only outcome of introducing disorder into the lattice of $1 \mathrm{~L}-\mathrm{MoS}_{2}$. Changes in the firstorder Raman peaks of $1 \mathrm{~L}-\mathrm{MoS}_{2}$ were also observed as the defect density increased, as shown in Figure 4. Although the peak fitting for the $E^{\prime}$ and $A^{\prime}{ }_{1}$ Raman peaks must be done with care due to additional peaks, as shown in Figure $4 \mathrm{~b}$, changes in both the peak positions and FWHMs are observed for both the $E^{\prime}$ and $A_{1}^{\prime}$ Raman peaks, as shown in Figures $4 \mathrm{c}$ and $4 \mathrm{~d}$ respectively. The Raman shift of the $E^{\prime}$ and $A^{\prime}{ }_{1}$ Raman peaks were found to decrease and increase respectively by several wavenumbers, effectively increasing the distance (a)

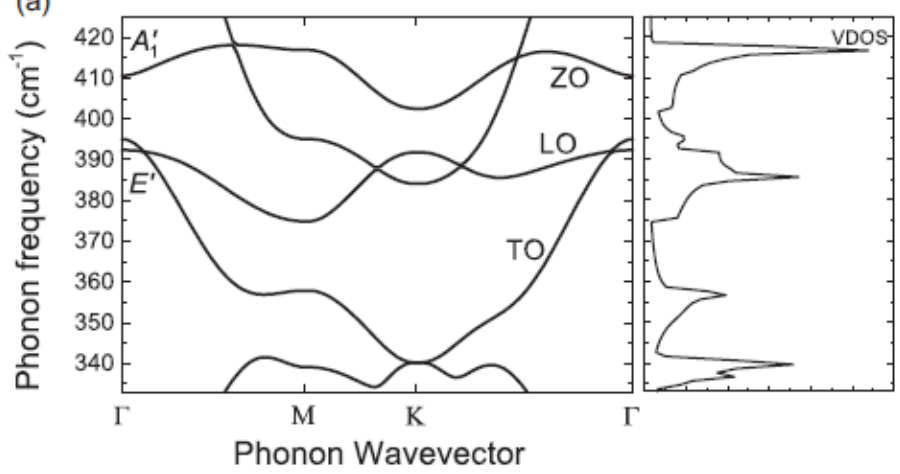

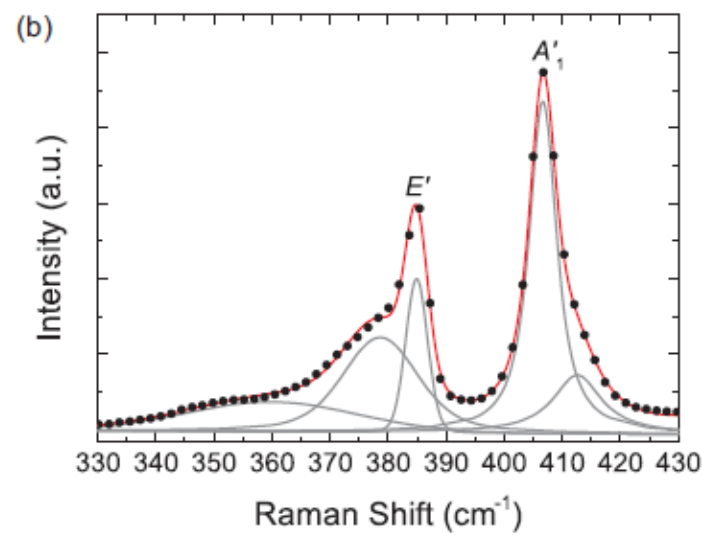
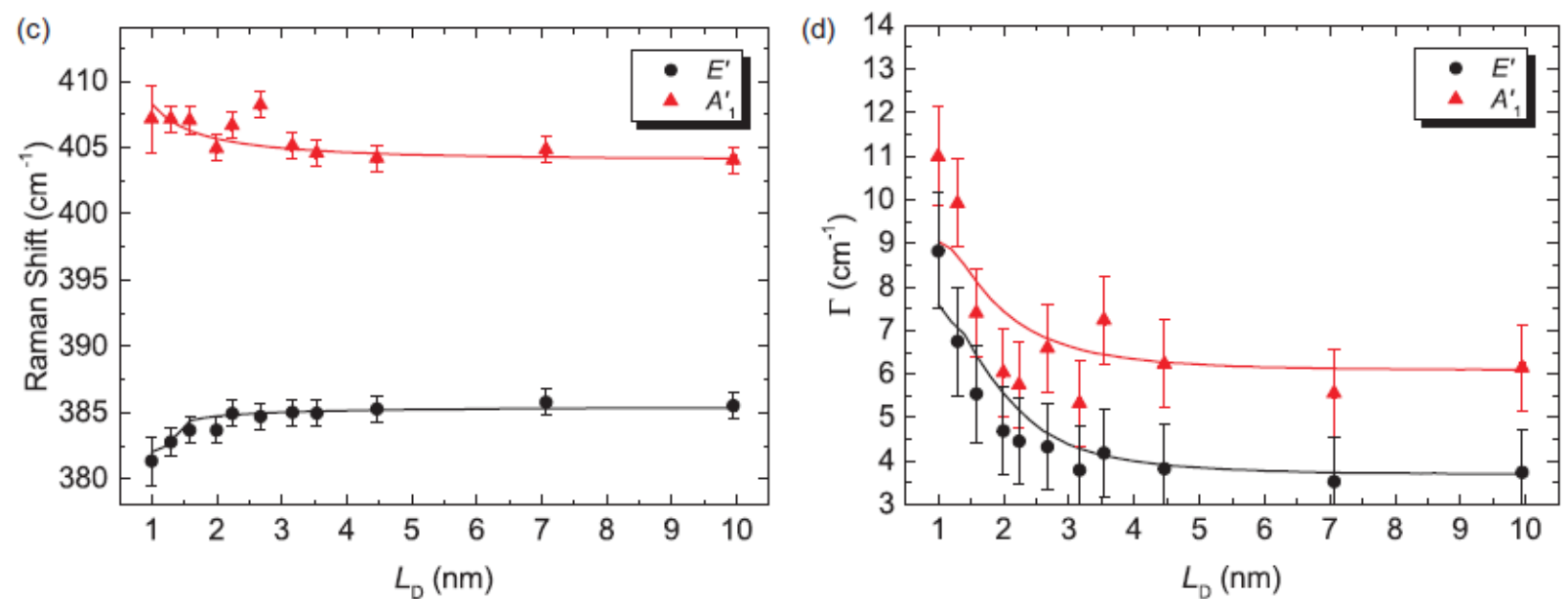

Figure 4. (a) Phonon dispersion and vibrational density of states of $1 \mathrm{~L}-\mathrm{MoS} 2$. (b) Close-up of the spectral region where the first-order peaks are located, for sample with $L_{\mathrm{D}}=2.2 \mathrm{~nm}$. The grey lines are the fitted Voigt peaks, and the red line is the cumulative spectrum from the Voigt fitting process. Symbols represent the experimental spectrum. (c) Position and (d) FWHM of $E^{\prime}$ and $A^{\prime}{ }_{1}$ as a function of $L_{D}$. Symbols refer to experimental data, continuous lines refer to the phonon confinement model described in the main text. The error bars for the peak position and $\Gamma$ represent the largest uncertainty present (the spectrometer resolution or the standard error from the fitting process), from [10] Copyright (2015) by the American Physical Society. 
between the peaks. This is of particular importance, as the distance between these two prominent peaks is typically used to determine the number of $\mathrm{MoS}_{2}$ layers present when performing Raman spectroscopy. This change in peak position, as well as the increase in FWHM for both Raman peaks shown in Figure 4d can be explained by a 'phonon confinement model'. When defects are introduced, these perturb the spatial translational invariance of the system and the phonon correlation length becomes finite, causing the breakdown of the fundamental selection rule, $\mathbf{q} \approx 0$.

These changes can be understood from the phonon dispersion curves in Figure 4a. Moving away from the $\Gamma$ point $(\mathbf{q} \sim 0)$ due to the greater defect densities, the LO and TO branches contributing to the $E^{\prime}$ peak show a downward trend, whilst the ZO branch corresponding to the $A^{\prime}{ }_{1}$ peak shows an upward trend. The effects of both doping and strain can be ruled out as the cause of the changes in the first-order peaks, as explained in detail in reference [10].

\section{Chemical Characterisation Removal of Polymer Residue}

and

The structural characterisation of graphene and other 2$\mathrm{D}$ materials, that is, the number of layers, lateral dimensions and level of disorder, is an obvious first step when trying to understand the material present. However, information on the chemical composition of the material is also critical and will affect many of the properties of interest for real-world applications. The chemical characterisation of any adventitious and undesired material present on real-world materials due to processing steps in large-scale production is also required. This is a particular issue for CVD-grown graphene and other 2-D materials which must be transferred from the metal growth substrate, typically using a sacrificial polymer film, further details on this type of transfer process are detailed in reference [12]. The removal of this polymer transfer layer is particularly problematic, as polymer residue remains on the surface of the 2-D material after the polymer film is 'removed' using wet-etching methods. This is also an issue when patterning these materials and producing a device, as these processes typically use photo-resist polymers. This residue has negative consequences with regard to the electrical properties of graphene and the reproducibility of any devices produced, due to the inhomogeneous distribution of undesired polymer contamination.

Secondary ion mass spectrometry (SIMS) is a powerful tool for characterising the chemical composition of materials, as well as surface contamination. Although not quantitative, SIMS can be performed alongside separate X-ray photoelectron spectroscopy (XPS) measurements in a complementary approach, as XPS is typically less sensitive but provides a quantitative assessment of the elements present.
SIMS measurements shown in Figure 5 reveal PMMA present on CVD-grown graphene after the graphene has been transferred to a substrate other than copper, upon which it was grown, and the sacrificial PMMA layer has been wet-etched using a solvent. The thickness of the PMMA residue present on the surface varied from a few nanometres up to $20 \mathrm{~nm}$, as shown in Figure $5 \mathrm{~b}$.
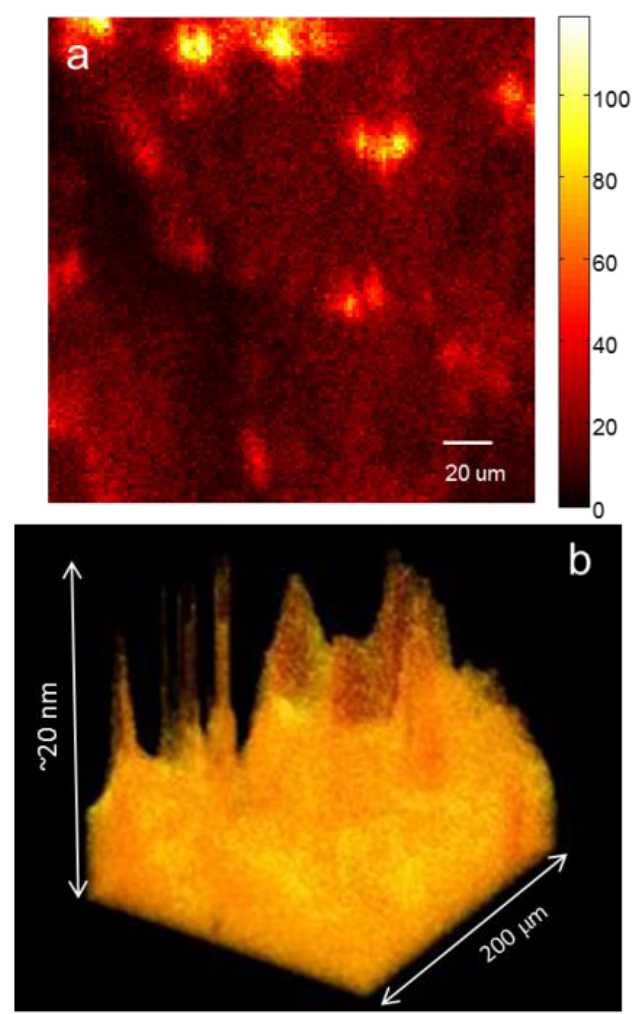

Figure 5. SIMS images of PMMA residue on a $200 \mu \mathrm{m} \times$ $200 \mu \mathrm{m}$ area of CVD-grown graphene. An image of the PMMA characteristic peak, integrated through the sputter depth, is shown in (a) and a 3D reconstruction of the PMMA overlayer is shown in (b), from [13].

This implies a more reproducible method is required to remove all of the sacrificial polymer layer. It must be possible to implement this process with current largescale semiconductor fabrication technology performed on the wafer-scale, whilst also not degrading the properties of the 2-D material by, for example, introducing defects into the crystal lattice.

Different techniques for removing organic contamination from graphene after wet-etching have previously been reported, including ultra-violet (UV) ozone treatment, argon plasma etching, vacuum annealing, annealing on a catalyst, contact-mode atomic force microscopy (AFM), and $\mathrm{CO}_{2}$ cluster jet conditioning. However, none of these methods are optimal for large-scale microelectronics manufacturing, may lead to defect formation in graphene or produce amorphous carbon on the surface.

Argon gas cluster ion beams (GCIBs) are already commercially available for the semiconductor industry for cleaning surfaces, and have also been shown to remove polymer material [14]. 
The Raman D- to G-peak intensity ratio of mechanically exfoliated 1LG bombarded by different argon GCIBs of varying size and energy as a function of ion dose (the number of argon cluster ions impinging the surface per area) is shown in Figure 6a. This reveals that the argon clusters can be varied in size and energy to drastically reduce the level of disorder introduced into a graphene single-layer [13]. Clusters with $1 \mathrm{eV} /$ atom or less required very significant ion dose levels to produce any significant level of disorder. Figure $6 \mathrm{~b}$, shows the level of disorder created in $1 \mathrm{LG}$ as a function of the equivalent amount of PMMA removed, to allow the determination of the optimal GCIB for limiting the damage to graphene whilst maximising the removal of PMMA. Comparing Figure $6 \mathrm{a}$ to Figure $2 \mathrm{c}$ from Section 2, the reduction in the disorder introduced into graphene is strikingly apparent for argon gas cluster bombardment compared to single ions, where an ion dose of 1 ion $/ \mathrm{nm}^{2}$ for $\mathrm{Mn}$, Bi or $\mathrm{Bi}_{3}$ ions is equivalent to $L_{\mathrm{D}}=1 \mathrm{~nm}$. This means that the argon gas clusters must typically impact the graphene surface many times (considering the size of an argon gas cluster compared to single ions in Section 2) for a chance to create a defect.
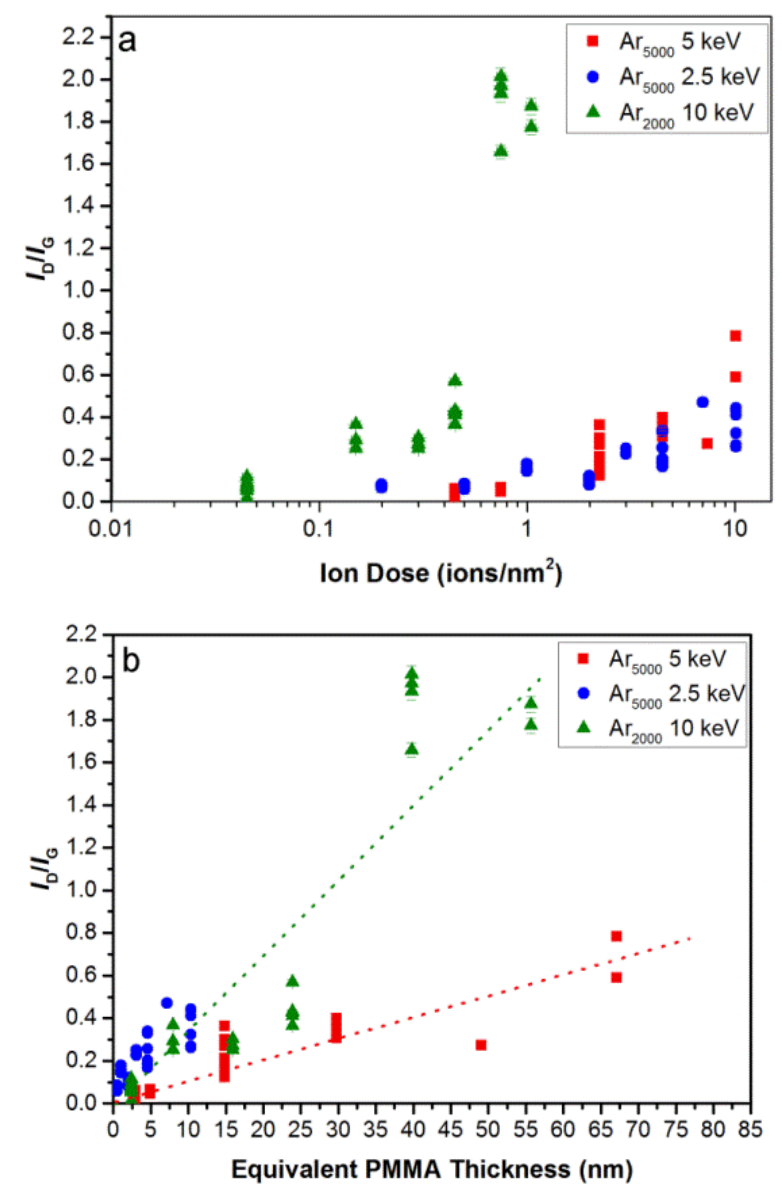

Figure 6. Ratio of Raman D- and G-peak intensities, as a function of Ar cluster ion dose for three GCIB conditions. (a) the intensity ratio as a function of ion dose and (b) the intensity ratio as a function of equivalent PMMA sputter depth, with broken lines included as a visual guide, from [13].
Figure 7 shows that the Ar GCIB can be used to remove polymer residue from CVD-grown graphene layers after they have been transferred to another substrate. SIMS imaging shows the large reduction in PMMA in the area dosed with the $\mathrm{Ar}_{5000}{ }^{+} 5 \mathrm{keV}$ clusters.
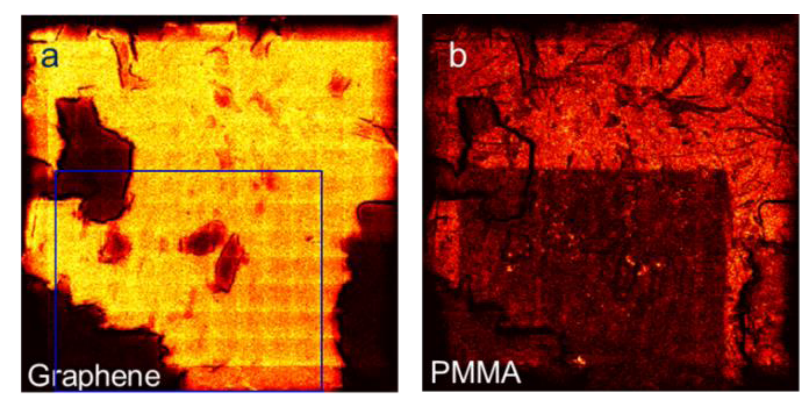

Figure $7.7 \mathrm{~mm} \times 7 \mathrm{~mm}$ ToF-SIMS image of a CVD-grown graphene sample after an area (outlined in blue) has been dosed with $1.00 \mathrm{ion} / \mathrm{nm}^{2}$ of $5 \mathrm{keV} \mathrm{Ar} \mathrm{Ar}_{500}$. (a) The location of the graphene, inferred based on differential charging of the sample, and (b) the PMMA signal image, from [13].

These results concur with previous work [14], which shows the sputtering yield of polymer/organic surfaces are 3-4 orders of magnitude greater than inorganic substrates for clusters with $1 \mathrm{eV} /$ atom. Figure $6 \mathrm{~b}$ shows that an argon gas cluster beam of 5000 atoms with $5 \mathrm{keV}$ energy is the optimal cluster for significant PMMA removal, whilst minimising the level of disorder introduced into the graphene lattice. This is an important consideration for this technique if the whole sample is to be dosed equally as, from Figure 5, the thickness of the PMMA residue is not uniform, thus some of the areas of graphene covered by less polymer would be dosed by the GCIB to ensure the areas with a thicker layer of PMMA are cleaned. Of course, for large-scale processing, a simultaneous Ar GCIB dosing and mass spectrometry monitoring system could be implemented to further minimise any damage to the graphene layers

\section{International Standardisation of Graphene and 2-D Materials}

Standardisation ensures technology is developed and commercialised in an open, safe and responsible manner, supporting regulation, environmental safety and commercialisation, thus enabling industry. Measurement standards are required to allow benchmarks to measure and compare different products in a reproducible way.

This is particularly important for the area of graphene as a fledgling industry emerges globally. These measurement standards will be crucial to allow the comparison of many different graphene/graphite materials produced worldwide, as well as for comparisons against other technologies. As with any nanotechnology, without the ability to accurately measure the material it will not be possible to adopt any Health and Safety standards. The consensus of the international community is also important and to this end international standardisation will be crucial for what will be a worldwide market. 
Although no standards have yet been published for graphene, there is currently activity in this area in both the International Organization of Standardization (ISO) and the International Electrotechnical Commission (IEC), in committees 'TC229 - Nanotechnologies' and 'TC113 - Nanotechnology standardization for electrical and electronic products and systems' respectively. These two committees have joint working groups in both the areas of 'Terminology and Nomenclature' and 'Measurement and Characterization', allowing close collaboration on terminology and measurement across a wide range of experts in different fields of nanotechnology. There are currently several standards in development:

- ISO/TS 80004-13 "Nanotechnologies - Vocabulary Part 13: Graphene and other two dimensional materials"

- ISO/TR 19733 "Matrix of characterization and measurement methods for Graphene"

- IEC/TS 62565-3-1 Ed. 1.0 "Nanomanufacturing Material specifications - Part 3-1: Graphene - Blank detail specification"

- IEC/TS 62565-3-2 Ed. 1.0 "Nanomanufacturing Material specifications - Part 3-2: Graphene - Detail specification for nano-ink"

- IEC/TS 62607-6-1 Ed. 1.0 "Nanomanufacturing - Key control characteristics - Part 6-1: Graphene - Electrical characterization"

- IEC/TS 62607-6-2 Ed. 1.0 "Nanomanufacturing - Key control characteristics - Part 6-2: Graphene - Evaluation of the number of layers of graphene"

- IEC/TS 62607-6-3 Ed. 1.0 "Nanomanufacturing - Key control characteristics - Part 6-3: Graphene Characterization of graphene domains and defects"

- IEC/TS 62607-6-4 Ed. 1.0 "Nanomanufacturing - Key control characteristics - Part 6-4: Graphene Conductance measurement using resonant cavity"

These standards are currently under development and are constantly evolving, with new work items also being discussed and planned. Some of these Technical Specifications have had more development than others and therefore the time-scale of publication will vary for these documents, with the hope that the first graphene Technical Specifications will be published in the next 12 years. Further details can be found at references $[15,16]$.

\section{Conclusions}

Graphene has many fantastic properties that have led to the prediction that this, and other 2-D materials, will be a disruptive technology for many different application areas. However, advances in metrology are needed to enable the commercialisation of these nanomaterials. The investigations at NPL into the measurement science of 2-D materials will be an important step in the future of graphene. Advances in the structural and chemical characterisation of graphene have been described, such as the level of disorder and the distinction of contamination, as well as developing new techniques to measure and improve these materials through, as an example, the removal of surface contamination. The continuation of international standardisation in the areas of graphene terminology and characterisation will also aid the emerging global graphene industry.

\section{Acknowledgements}

The author would like to acknowledge the National Measurement Office (NMO) for funding through the Innovation, Research and Development (IRD) programme (Project No.118616) and Dr Andrew Wain for discussions related to the manuscript.

\section{References}

1. K. S. Novoselov, A. K. Geim, S. V. Morozov, D. Jiang, Y. Zhang, S. V. Dubonos, I. V. Grigorieva, A. A. Firsov, Science, 306, 666 (2004)

2. A. C. Ferrari et al., Nanoscale, 7, 4598 (2015)

3. A. Tzalenchuk, S. Lara-Avila, A. Kalaboukhov, S. Paolillo, M. Syväjärvi, R. Yakimova, O. Kazakova, T. J. B. M. Janssen, V. Fal'ko and S. Kubatkin, Nat. Nanotechnol. 5, 186 (2010)

4. F. Banhart, J. Kotakoski and A. V. Krasheninnikov, ACS Nano, 5, 26 (2011)

5. S. C. O’Hern, M. S. H. Boutilier, J-C. Idrobo, Y. Song, J. Kong, T. Laoui, M. Atieh, and R. Karnik, Nano. Lett., 141234 (2014)

6. M. M. Lucchese, F. Stavale, E. H. Martins Ferreira, C. Vilani, M. V. O. Moutinho, R. B. Capaz, C. A. Achete and A. Jorio, Carbon, 48, 1592 (2010)

7. L. G. Cancado, A. Jorio, E. H. M. Ferreira, F. Stavale, C. A. Achete, R. B. Capaz, M. V. O. Moutinho, A. Lombardo, T. S. Kulmala and A. C. Ferrari, Nano Lett., 11, 3190 (2011)

8. A. J. Pollard, B. Brennan, H. Stec, B. J. Tyler, M. P. Seah, I. S. Gilmore, and D. Roy, App. Phys. Lett., 106, 253107 (2014)

9. M. Xu, T. Lian, M. Shi and H. Chen, Chem. Rev. 113, 3766 (2013)

10. S. Mignuzzi, A. J. Pollard, N. Bonini, B. Brennan, I. S. Gilmore, M. A. Pimenta, D. Richards, and D. Roy, Phys. Rev. B, 91, 195411 (2015)

11. K. S. Novoselov, D. Jiang, F. Schedin, T. J. Booth, V. V. Khotkevich, S. V. Morozov, and A. K. Geim, Proc. Natl. Acad. Sci. U.S.A., 102, 10451 (2015)

12. A. J. Pollard, R. R. Nair, S. N. Sabki, C. R. Staddon, L. M. A. Perdigao, C. H. Hsu, J. M. Garfitt, S. Gangopadhyay, H. F. Gleeson, A. K. Geim and P. H. Beton, J. Phys. Chem. C, 112, 15439 (2009)

13. B. J. Tyler, B. Brennan, H. Stec , T. Patel, L. Hao, I. S Gilmore, and A. J. Pollard, J. Phys. Chem. C, Just Accepted Manuscript, DOI: 10.1021/acs.jpcc.5b03144 (2015)

14. M. P Seah, J. Phys. Chem. C, 117, 12622 (2013)

15. www.iso.org/iso/home/store/catalogue tc/catalogue tc browse.htm? commid $=381983$

16. www.iec.ch/dyn/www/f?p=103:30:0::::FSP_ORG ID,FSP LANG ID:1315,25 